\title{
Ultrasound-guided percutaneous
} cryoneurolysis providing postoperative analgesia lasting many weeks following a single administration: a replacement for continuous peripheral nerve blocks?

\section{-a case report-}

\author{
Brian M. Ilfeld ${ }^{1}$, Rodney A. Gabriel ${ }^{1}$, and Andrea M. Trescot ${ }^{2}$ \\ ${ }^{1}$ Department of Anesthesiology, University of California San Diego, San Diego, CA, ${ }^{2}$ Pain and Headache Center, \\ Eagle River, AK, USA
}

\begin{abstract}
Cryoneurolysis entails using low temperatures to reversibly ablate nerves, with a subsequent analgesia duration measured in weeks or months. Previously, clinical applications for acute pain were limited because treatment originally required exposing the target nerve surgically. However, three developments have now made it possible to provide prolonged postoperative analgesia by cryoneurolysis: 1) new portable, hand-held cryoneurolysis devices, 2) ultrasound machine proliferation, and, 3) anesthesiologists trained in ultrasound-guided peripheral nerve block administration. This report is the first to describe the use of a single preoperative administration of ultrasound-guided percutaneous cryoneurolysis to provide multiple weeks of analgesia following shoulder rotator cuff repair and total knee arthroplasty. Considering the significant benefits of cryoanalgesia relative to continuous peripheral nerve blocks (e.g., lack of catheter/pump care, extremely long duration), this analgesic modality may be a practical alternative for the treatment of prolonged post-surgical pain in a select group of surgical patients.
\end{abstract}

Key Words: Cryoablation, Cryoanalgesia, Cryoneuroanalgesia, Perioperative analgesia, Postoperative analgesia.

Corresponding author: Brian M. Ilfeld, M.D., M.S.

Department of Anesthesiology, University of California San Diego,

200 West Arbor Drive, MC 8770, San Diego, CA 92103-8770, USA

Tel: 1-858-657-7030, Fax: 1-858-683-2003

Email: bilfeld@ucsd.edu

ORCID: https://orcid.org/0000-0002-6144-3273

Received: December 8, 2016.

Revised: December 21, 2016.

Accepted: December 21, 2016.

Korean J Anesthesiol 2017 October 70(5): 567-570

https://doi.org/10.4097/kjae.2017.70.5.567
Some surgical procedures result in prolonged, moderate-tosevere postoperative pain that is often difficult to control. Continuous peripheral nerve blocks are often effective analgesics, but are usually limited to a few days due to infection risk, and bring a host of possible complications, such as catheter dislocation and leakage, infusion pump malfunction, and the burden of carrying a local anesthetic reservoir [1]. One possible alternative analgesic modality is cryoneurolysis: using extremely low temperatures to reversibly ablate nerves, with a subsequent analgesia duration measured in weeks or months [2]. The technique has been in use for decades to treat chronic pain, but new advances

(c) This is an open-access article distributed under the terms of the Creative Commons Attribution Non-Commercial License (http://creativecommons.org/ licenses/by-nc/4.0/), which permits unrestricted non-commercial use, distribution, and reproduction in any medium, provided the original work is properly cited. 
in cryoneurolysis equipment and ultrasound training raise the possibility of using cryoneurolysis to replace continuous peripheral nerve blocks to treat acute postoperative pain [3]. This report is the first to describe the use of a single preoperative administration of ultrasound-guided percutaneous cryoneurolysis to provide multiple weeks of analgesia following shoulder rotator cuff repair and total knee arthroplasty in five patients.

\section{Case Report}

Five patients undergoing orthopedic surgery of the shoulder $(\mathrm{n}=2)$ and knee $(\mathrm{n}=3)$ were offered, and consented to, preoperative administration of ultrasound-guided percutaneous cryoneurolysis for postoperative analgesia. The university's Institutional Review Board (University of California San Diego, San Diego, CA, USA) waives review requirements for case reports and short series, but all patients provided verbal consent for publication of these case reports. Additionally, all patients provided written informed consent for the cryoneurolysis procedures themselves, including with respect to the potential risks of cryoablation (unrelated to this report). Patients were positioned supine (knee) or prone with the chest supported on pillows and ipsilateral arm hanging over the side of the gurney (shoulder). American Society of Anesthesiologist standard monitors were used and the cryoprobe entry site was prepared with chlorhexidine gluconate.

A portable ultrasound (SonoSite M-Turbo, Bothell, WA, USA) and 13-6 MHz linear ultrasound transducer (HFL38; SonoSite) within a sterile sleeve were used to image the target nerves in a short-axis (cross sectional) view: the suprascapular nerve just superior to the suprascapular notch (shoulder) [4] or the infrapatellar branch of the saphenous nerve distal to its origin from the femoral nerve (knee) [5,6]. A 20 gauge $(G)$ angiocatheter was inserted through a lidocaine $1 \%$ (with epinephrine $1: 200,000$ for hemostasis) skin wheal immediately caudal to the transducer, and lidocaine $2 \%(1 \mathrm{ml})$ was deposited adjacent to the target nerve under ultrasound guidance. If accurate angiocatheter positioning was confirmed by sensory changes within the distribution of the target nerve occurring within $15 \mathrm{~min}$, the

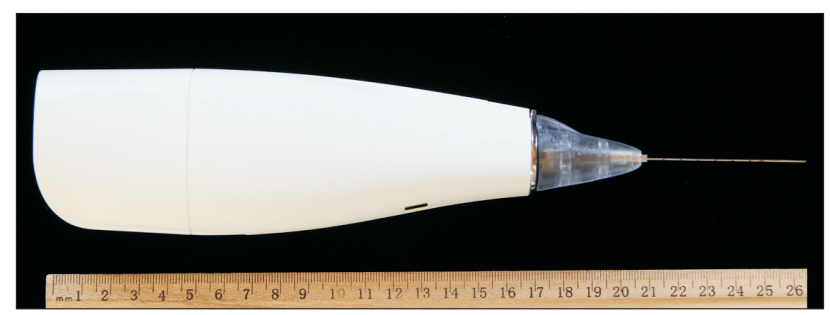

Fig. 1. A portable cryoneurolytic device with a $5.5-\mathrm{cm}, 22$ gauge cryoprobe (Iovera Focused Cold Therapy, Myoscience, Freemont, CA, USA). stylet of the angiocatheter was withdrawn to allow insertion of the cryoneurolysis probe.

All procedures were executed using a $5.5-\mathrm{cm}, 22-\mathrm{G}$, singleuse cryoneurolysis probe (Smart Tip; Myoscience, Freemont, CA, USA) inserted under ultrasound guidance through the angiocatheter because the probe's closed tip is somewhat blunt [3]. A portable, handheld cryoablation device (Iovera Focused Cold Therapy; Myoscience Freemont, CA, USA)) was used to deliver nitrous oxide $\left(\mathrm{N}_{2} \mathrm{O}\right)$ through the center of the probe, using two cycles of 3-min freezing to $-70^{\circ} \mathrm{C}$, separated by a 2 -min defrost (Fig. 1). No patient reported discomfort during the cryoneurolysis itself, likely due to the previously injected lidocaine. A general anesthetic was administered for the surgical procedure.

All five patients experienced excellent postoperative analgesia with pain scores consistently $<2$ on a $0-10$ numeric rating scale, and required significantly less opioid for a decreased period of time relative to historic controls $[7,8]$. For the shoulder rotator cuff repair patients, there was full return of motor function by approximately 2-3 weeks following the original procedure. For patients having knee surgery, no gross motor deficit was noted, relative to historic controls, and skin sensation returned within 4 months. There was no adverse event related to the cryoneurolysis.

\section{Discussion}

The cases discussed here suggest that cryoneurolysis (also known as "cryoanalgesia" or "cryoneuroanalgesia") may be a viable alternative to treating prolonged postoperative pain in a select cohort of patients (possibly particularly beneficial in patients at high risk for persistent post-surgical pain) $[9,10]$. While cryoneurolysis has been used for decades to provide relief from chronic nerve pain [11], it was not until the 1980s that cryoneurolysis was applied to surgically exposed nerves to provide postoperative analgesia [12]. Probe technology advances have enabled percutaneous insertion and reports of its use treating chronic pain increased subsequently $[2,13]$, with a single retrospective report describing use to treat an acute pain state [14]. However, the advent of ultrasound guidance now enables extremely precise application to virtually any peripheral nerve [3]. The present report is, to our knowledge, the first to describe the preoperative use of ultrasound-guided percutaneous cryoneurolysis to provide multiple weeks of postoperative analgesia.

A modern cryoneurolytic probe creates extremely cold temperatures by passing a gas down the shaft at high pressure and through a small opening into a closed tip at low pressure, resulting in absorption of heat due to the Joule-Thomson effect (Fig. 2) [3]. The gas is evacuated through an internal tube within the shaft so that no gas makes contact with, or remains within, the patient. An ice ball forms at the tip of the probe, inducing Wallerian degeneration distal to the point of ablation and result- 


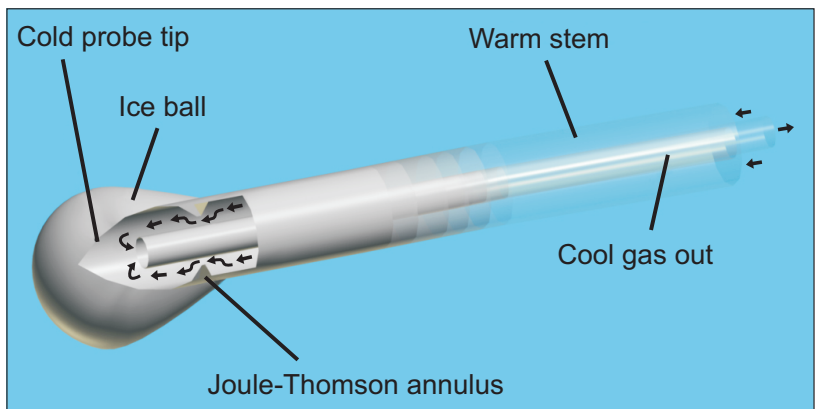

Fig. 2. A modern cryoneurolysis probe ("cannula") produces extremely cold temperatures at its tip due to the Joule-Thomson effect, resulting from gas flowing from a high- to a low-pressure chamber (used with permission).

ing in a complete conduction block. Importantly, the endo-, peri-, and epineurium remain intact, allowing neural regrowth distally at $1-2 \mathrm{~mm} /$ day without the risk of neuroma formation. Block duration is dependent on multiple factors, such as the distance from the ablation to the terminal nerve ending, surrounding tissue temperature, and nerve diameter. The result is that the duration of analgesia is highly variable and often unpredictable, but is usually measured in weeks or months. Whether block duration could be better controlled by altering factors such as the freeze and/or thaw duration, or number of freeze-thaw cycles, requires further research.

Contraindications include Raynaud's syndrome, cryoglobulinemia, cold urticaria, bleeding disorders, localized infection, and anticoagulation. Risks include depigmentation, hyperpigmentation, alopecia in the hairline, and "frostbite" of the skin if the ice ball encompasses the dermis, as well as bruising and bleeding. Three clinical trials involving cryoneurolysis of intercostal nerves through thoracotomy incisions reported an increased risk of postoperative neuropathic pain, while many others found no such association [12].

The main limiting factors regarding the use of cryoneurolysis to treat postoperative pain are the complete sensory and motor block combined with the prolonged and often unpredictable duration of action. Prolonged sensory and/or motor blockade may not be desirable or safe in many circumstances. Specific acute pain applications (nerves) possibly amenable to cryoneurolysis are total shoulder arthroplasty and rotator cuff repair (suprascapular nerve), total knee arthroplasty and anterior cruciate ligament repair (infrapatellar branch of the saphenous nerve and anterior femoral cutaneous nerve), rib fractures and thoracotomy (intercostal), herniorrhaphy (intercostal/ilioinguinal), tonsillectomy (glossopharyngeal), iliac crest bone grafts (superior cluneal and/or subcostal nerves), and amputations and burns (any involved terminal nerve). Importantly, high-quality randomized, controlled clinical trials are necessary to gauge the benefit-to-risk ratio and optimize techniques/equipment prior to widespread adoption of perioperative cryoneurolysis, and informed consent should include the potential risk of neuropathic pain, at least until this issue has been resolved [12]. Nevertheless, the cases presented in this report demonstrate the possibility of applying ultrasound-guided percutaneous cryoneurolysis to provide analgesia following surgical procedures.

\section{Acknowledgments}

The authors would like to thank Elan Ilfeld for his rendering of Fig. 2.

\section{Conflict of Interest}

The cryoneurolysis device, probes, and nitrous oxide canisters used in these case reports were provided by the manufacturer (Myoscience, Fremont, California, United States). This company had no input into any aspect of these cases or manuscript preparation. The content of this article is solely the responsibility of the authors and does not necessarily represent the official views of this company.

\section{References}

1. Ilfeld BM. Continuous peripheral nerve blocks: a review of the published evidence. Anesth Analg 2011; 113: 904-25.

2. Trescot AM. Cryoanalgesia in interventional pain management. Pain Physician 2003; 6: 345-60.

3. Ilfeld BM, Preciado J, Trescot AM. Novel cryoneurolysis device for the treatment of sensory and motor peripheral nerves. Expert Rev Med Devices 2016; 13: 713-25.

4. Elsharkawy HA, Abd-Elsayed AA, Cummings KC 3rd, Soliman LM. Analgesic efficacy and technique of ultrasound-guided suprascapular nerve catheters after shoulder arthroscopy. Ochsner J 2014; 14: 259-63.

5. Lundblad M, Forssblad M, Eksborg S, Lönnqvist PA. Ultrasound-guided infrapatellar nerve block for anterior cruciate ligament repair: a prospective, randomised, double-blind, placebo-controlled clinical trial. Eur J Anaesthesiol 2011; 28: 511-8.

6. Lundblad M, Kapral S, Marhofer P, Lönnqvist PA. Ultrasound-guided infrapatellar nerve block in human volunteers: description of a novel technique. Br J Anaesth 2006; 97: 710-4.

7. Ilfeld BM, Mariano ER, Girard PJ, Loland VJ, Meyer RS, Donovan JF, et al. A multicenter, randomized, triple-masked, placebo-controlled 
trial of the effect of ambulatory continuous femoral nerve blocks on discharge-readiness following total knee arthroplasty in patients on general orthopaedic wards. Pain 2010; 150: 477-84.

8. Ilfeld BM, Morey TE, Wright TW, Chidgey LK, Enneking FK. Continuous interscalene brachial plexus block for postoperative pain control at home: a randomized, double-blinded, placebo-controlled study. Anesth Analg 2003; 96: 1089-95.

9. Kehlet H, Jensen TS, Woolf CJ. Persistent postsurgical pain: risk factors and prevention. Lancet 2006; 367: 1618-25.

10. Ilfeld BM, Madison SJ, Suresh PJ, Sandhu NS, Kormylo NJ, Malhotra N, et al. Persistent postmastectomy pain and pain-related physical and emotional functioning with and without a continuous paravertebral nerve block: a prospective 1-year follow-up assessment of a randomized, triple-masked, placebo-controlled study. Ann Surg Oncol 2015; 22: 2017-25.

11. Gage AA. History of cryosurgery. Semin Surg Oncol 1998; 14: 99-109.

12. Khanbhai M, Yap KH, Mohamed S, Dunning J. Is cryoanalgesia effective for post-thoracotomy pain? Interact Cardiovasc Thorac Surg 2014; 18: 202-9.

13. Moesker AA, Karl HW, Trescot AM. Treatment of phantom limb pain by cryoneurolysis of the amputated nerve. Pain Pract 2014; 14: 52-6.

14. Dasa V, Lensing G, Parsons M, Harris J, Volaufova J, Bliss R. Percutaneous freezing of sensory nerves prior to total knee arthroplasty. Knee 2016; 23: 523-8. 